MATHEMATICS OF COMPUTATION

Volume 76, Number 259, July 2007, Pages 1119-1140

S 0025-5718(07)01951-5

Article electronically published on January 24, 2007

\title{
CONTINUOUS INTERIOR PENALTY $h p$-FINITE ELEMENT METHODS FOR ADVECTION AND ADVECTION-DIFFUSION EQUATIONS
}

\author{
ERIK BURMAN AND ALEXANDRE ERN
}

\begin{abstract}
A continuous interior penalty $h p$-finite element method that penalizes the jump of the gradient of the discrete solution across mesh interfaces is introduced. Error estimates are obtained for advection and advection-diffusion equations. The analysis relies on three technical results that are of independent interest: an $h p$-inverse trace inequality, a local discontinuous to continuous $h p$-interpolation result, and $h p$-error estimates for continuous $L^{2}$-orthogonal projections.
\end{abstract}

\section{INTRODUCTION}

Interior penalty procedures for finite element methods utilizing continuous functions have been introduced in the pioneering works of Babuška and Zlámal 3 for the biharmonic operator and of Douglas and Dupont 12 for second-order elliptic and parabolic problems. The common feature of these methods consists of penalizing the jump of the gradient of the discrete solution at mesh interfaces, but the motivations behind [3] and [12] are different. The goal pursued in [3] was to weakly enforce $C^{1}$-continuity. Because of a non-consistency in the formulation, a superpenalty procedure had to be applied, leading to suboptimal convergence rates. The subsequent work of Baker 4, valid for general $2 m$ th order coercive operators, designed a consistent interior penalty method, utilizing discontinuous functions, that was shown to be optimally convergent.

The motivation behind the work of Douglas and Dupont was different, namely to keep continuous finite element methods because they were standard for elliptic problems and, at the same time, to cope with the difficulties encountered by such methods in problems where the first-order (advection) terms dominate the second-order (diffusion) terms. However, one of the main issues at stake, namely the robustness of the error estimates with respect to the cell Péclet numbers, was not addressed in 12. This issue has only been addressed quite recently for linear finite elements, namely in the work of Burman and Hansbo in 2004 [9]. A unified framework for the convergence analysis of both continuous and nonconforming (Crouzeix-Raviart) finite elements with interior penalty, still in the linear case, has been proposed in [5], and a nonlinear shock-capturing term based on gradient jumps that rigorously

Received by the editor January 24, 2005 and, in revised form, March 25, 2006.

2000 Mathematics Subject Classification. Primary 65N30, 65N12, 65N15, 65D05, 65N35.

Key words and phrases. Continuous interior penalty, $h p$-finite element method, convectiondiffusion, $h p$-interpolation and projection, $h p$-inverse trace inequality.

(C)2007 American Mathematical Society Reverts to public domain 28 years from publication 
guarantees a discrete maximum principle for advection-diffusion-reaction problems has been investigated in [6].

The goal of this paper is to present, for the first time, an $h p$-convergence analysis for a high-order Continuous Interior Penalty (CIP) finite element method applied to advection and (advection-dominated) advection-diffusion equations. The $h p$-version of the finite element method was introduced in the 1980s following the analysis presented by Babuška and Dorr 1 and by Babuška and Suri 2 for secondorder elliptic problems. See also 25] for a review of theoretical background and applications of $p$ - and $h p$-finite element methods. For advection and (advectiondominated) advection-diffusion equations, $h p$-finite element approximations have been investigated by Houston, Schwab, and Süli for continuous finite elements with streamline diffusion stabilization and for interior penalty discontinuous finite elements [18, 20, 19], leading, respectively, to the so-called $h p$-Streamline Diffusion (SD) method and the $h p$-Discontinuous Galerkin (DG) method. These are, to date, the two established methods for the $h p$-finite element approximation of advection and (advection-dominated) advection-diffusion equations.

The $h p$-CIP finite element method investigated in this paper penalizes the jump of the gradient of the discrete solution at mesh interfaces. One advantage with respect to both the $h p$-SD method and the $h p$-DG method is that the stabilization parameter is independent of the diffusion coefficient. This can be important in nonlinear problems where this coefficient depends on the discrete solution. The other advantage with respect to the $h p$-DG method is that the $h p$-CIP method requires less degrees of freedom (though only marginally less for high-order polynomials). The other advantage with respect to the $h p$-SD method is that the $h p$-CIP method leads to a single, symmetric stabilization term. In the $h p$-SD method, the stabilization terms involve couplings with the second-order term, the source term, and the time-derivative. This can pose problems when approximating stiff problems. The price to be paid for these advantages in the $h p$-CIP method is a slight sub-optimality (proportional to $p^{\frac{1}{4}}$ ) in the error estimate and, compared with the $h p$-SD method, a less compact discretization stencil. However, the $h p$-CIP method has optimal convergence properties in the diffusion-dominated regime, as opposed to the $h p$-DG method where a factor of $p^{\frac{1}{2}}$ is lost.

This paper is organized as follows. Section 2 introduces the discrete setting and the $h p$-CIP finite element method to approximate a first-order reaction-advection equation. Section 3 contains three technical results, that are of independent interest, for tensor-product finite elements. We prove an $h p$-inverse trace inequality, a local discontinuous to continuous $h p$-interpolation result, and $h p$-error estimates for continuous $L^{2}$-orthogonal projections. Section 4 presents the convergence analysis of the method in the spirit of Strang's Second Lemma. Section 5 discusses the extension of the results derived in Sections 3 and 4 to simplicial finite elements. Section 6 investigates the extension of the results derived for tensor-product finite elements to advection-diffusion equations. Section 7 draws some conclusions.

\section{Continuous interior Penalty finite Element methods}

Let $\Omega$ be an open bounded and connected set in $\mathbb{R}^{d}, d \leq 3$, with Lipschitz boundary $\partial \Omega$ and outer normal $n$, let $\beta \in\left[W^{1, \infty}(\Omega)\right]^{d}$ be a vector field, and let $\sigma \in L^{\infty}(\Omega)$. Let $f \in L^{2}(\Omega)$, let $\partial \Omega^{ \pm}=\{x \in \partial \Omega ; \pm \beta(x) \cdot n(x)>0\}$, and consider 
the problem

$$
\left\{\begin{aligned}
\sigma u+\beta \cdot \nabla u & =f \\
\left.u\right|_{\partial \Omega^{-}} & =0 .
\end{aligned}\right.
$$

Define $W=\left\{w \in L^{2}(\Omega) ; \beta \cdot \nabla w \in L^{2}(\Omega)\right\}$. Provided $\partial \Omega^{+}$and $\partial \Omega^{-}$are wellseparated [14] (see also [21]), functions in $W$ have traces in $L^{2}(\partial \Omega ;|\beta \cdot n|)$, that is, in the space of square integrable functions on $\partial \Omega$ for the surface measure $|\beta \cdot n| \mathrm{d} \lambda$ where $\mathrm{d} \lambda$ is the surface Lebesgue measure. Consider the operator $A: W \ni w \mapsto$ $\sigma w+\beta \cdot \nabla w \in L^{2}(\Omega)$. Henceforth, it is assumed that there is $\sigma_{0}>0$ such that

$$
\sigma-\frac{1}{2} \nabla \cdot \beta \geq \sigma_{0}, \quad \text { a.e. in } \Omega .
$$

Then, letting $V=\left\{w \in W ;\left.w\right|_{\partial \Omega^{-}}=0\right\}, A: V \rightarrow L^{2}(\Omega)$ is an isomorphism, i.e., (11) is well-posed; see, e.g., 15, 14.

Let $\mathcal{K}$ be a subdivision of $\Omega$ into non-overlapping cells $\{\kappa\}$. For $\kappa \in \mathcal{K}, h_{\kappa}$ denotes its diameter. Set $h=\max _{\kappa \in \mathcal{K}} h_{\kappa}$. Assume that (i) $\mathcal{K}$ covers $\bar{\Omega}$ exactly, (ii) $\mathcal{K}$ does not contain any hanging nodes, and (iii) $\mathcal{K}$ is quasi-uniform in the sense that there exists a constant $\rho>0$, independent of $h$, such that $\rho h \leq \min _{\kappa \in \mathcal{K}} h_{\kappa}$. Each $\kappa \in \mathcal{K}$ is assumed to be an affine image of the unit hypercube $\widehat{\kappa}=[-1,1]^{d}$, i.e., $\kappa=T_{\kappa}(\widehat{\kappa})$. Let $\mathcal{F}$ denote the set of interior faces $((d-1)$-manifolds) of the mesh. For $F \in \mathcal{F}, h_{F}$ denotes its diameter.

Let $p \geq 1$ and let $\mathbb{Q}_{p, d}(\widehat{\kappa})$ be the space of polynomials of degree at most $p$ in each variable. Introduce the finite element spaces

$$
\begin{aligned}
V_{h}^{p} & =\left\{v_{h} \in C^{0}(\bar{\Omega}) ; \forall \kappa \in \mathcal{K},\left.v_{h}\right|_{\kappa} \circ T_{\kappa} \in \mathbb{Q}_{p, d}(\widehat{\kappa})\right\}, \\
W_{h}^{p} & =\left\{w_{h} \in L^{2}(\Omega) ; \forall \kappa \in \mathcal{K},\left.w_{h}\right|_{\kappa} \circ T_{\kappa} \in \mathbb{Q}_{p, d}(\widehat{\kappa})\right\} .
\end{aligned}
$$

For a subset $R \subset \Omega,(\cdot, \cdot)_{R}$ denotes the $L^{2}(R)$-scalar product, $\|\cdot\|_{R}=(\cdot, \cdot)_{R}^{1 / 2}$ the corresponding norm, and $\|\cdot\|_{s, R}$ the $H^{s}(R)$-norm. For $s \geq 1$, let $H^{s}(\mathcal{K})$ be the space of piecewise $H^{s}$ functions. For $v \in H^{2}(\mathcal{K})$ and an interior face $F=\kappa_{1} \cap \kappa_{2}$, where $\kappa_{1}$ and $\kappa_{2}$ are two distinct elements of $\mathcal{K}$ with respective outer normals $n_{1}$ and $n_{2}$, introduce the (scalar-valued) jump $[\nabla v \cdot n]_{F}=\left.\nabla v\right|_{\kappa_{1}} \cdot n_{1}+\left.\nabla v\right|_{\kappa_{2}} \cdot n_{2}$ (the subscript $F$ is dropped when there is no ambiguity). Similarly, for $v \in H^{1}(\mathcal{K})$, define the (scalar-valued) jump $[v]_{F}=\left.v\right|_{\kappa_{1}}-\left.v\right|_{\kappa_{2}}$ (the arbitrariness in the sign of $[v]_{F}$ can be avoided by considering the vector-valued jump $[v]_{F}=\left.v\right|_{\kappa_{1}} n_{1}+\left.v\right|_{\kappa_{2}} n_{2}$; nothing that is stated hereafter depends on this arbitrariness).

On $H^{1}(\Omega) \times H^{1}(\Omega)$ define the standard Galerkin bilinear form

$$
a(v, w)=((\sigma-\nabla \cdot \beta) v, w)_{\Omega}-(v, \beta \cdot \nabla w)_{\Omega}+(\beta \cdot n v, w)_{\partial \Omega^{+}},
$$

and on $H^{q}(\mathcal{K}) \times H^{q}(\mathcal{K}), q>\frac{3}{2}$, define the $h p$-CIP bilinear form

$$
j(v, w)=\sum_{F \in \mathcal{F}} \frac{h_{F}^{2}}{p^{\alpha}} \beta_{\infty, F}([\nabla v \cdot n],[\nabla w \cdot n])_{F},
$$

where $\beta_{\infty, F}:=\left\|\beta \cdot n_{F}\right\|_{L^{\infty}(F)}$ and $n_{F}$ is a unit normal vector to $F$ (its orientation is irrelevant). Since $W^{1, \infty}(\Omega) \subset C^{0}(\bar{\Omega})$, the field $\beta$ is continuous by assumption and, therefore, the quantity $\beta \cdot n_{F}$ is single-valued on all interior faces. The exponent $\alpha$ will be determined by the $h p$-convergence analysis in 4 see (41).

The $h p$-CIP finite element approximation to (1) consists of finding $u_{h} \in V_{h}^{p}$ such that

$$
a\left(u_{h}, v_{h}\right)+j\left(u_{h}, v_{h}\right)=\left(f, v_{h}\right)_{\Omega}, \quad \forall v_{h} \in V_{h}^{p} .
$$


For $v \in H^{q}(\mathcal{K}), q>\frac{3}{2}$, consider the norm

$$
\|v\|_{a, j}^{2}=\left\|\sigma_{0}^{\frac{1}{2}} v\right\|_{\Omega}^{2}+\frac{1}{2}\left\||\beta \cdot n|^{\frac{1}{2}} v\right\|_{\partial \Omega}^{2}+j(v, v) .
$$

The well-posedness of the approximate problem (7) results from the following.

Lemma 2.1 (Coerciveness). For all $v \in H^{q}(\mathcal{K}), q>\frac{3}{2}, a(v, v)+j(v, v) \geq\|v\|_{a, j}^{2}$.

Proof. Straightforward verification using the divergence formula.

\section{TEChNiCAL RESUlts}

Henceforth, $c$ denotes a generic constant, independent of $p$ and $h$; its actual value can change at each occurrence.

3.1. $h p$-trace inequalities. Let $\left\{g_{j}\right\}_{0 \leq j \leq p}$ be the Gauß-Lobatto nodes in the unit interval $[-1,1]$. Set $I_{p, d}=\{0, \ldots, p\}^{d}$ and $I_{p, d}^{0}=\{1, \ldots, p-1\}^{d}$. For a multi-index $(i)=\left(i_{1}, \ldots, i_{d}\right) \in I_{p, d}$, the tensor-product Gauß-Lobatto node $a_{\widehat{\kappa},(i)}$ in the unit hypercube $\widehat{\kappa}$ is the point with coordinates equal to $\left(g_{i_{1}}, \ldots, g_{i_{d}}\right)$.

Let $\kappa \in \mathcal{K}$. Introduce the tensor-product Gauß-Lobatto nodes in $K$ such that $a_{\kappa,(i)}=T_{\kappa}\left(a_{\widehat{\kappa},(i)}\right)$ for all $(i) \in I_{p, d}$ and define the space

$$
\mathbb{Q}_{p, d}^{0}(\kappa)=\left\{v \in \mathbb{Q}_{p, d}(\kappa) ; \forall(i) \in I_{p, d}^{0}, v\left(a_{\kappa,(i)}\right)=0\right\} .
$$

In other words, $\mathbb{Q}_{p, d}^{0}(\kappa)$ is the subspace of $\mathbb{Q}_{p, d}(\kappa)$ spanned by those polynomials that vanish at all the interior tensor-product Gauß-Lobatto nodes in $\kappa$. The following result is stated in arbitrary space dimension since it can be of independent interest.

Lemma 3.1. The following trace and inverse trace inequalities hold:

$$
\begin{aligned}
& \forall v \in \mathbb{Q}_{p, d}(\kappa), \quad\|v\|_{\partial \kappa} \leq\left(\frac{p(p+1)}{2}\left(2+\frac{1}{p}\right)^{d} \frac{\operatorname{meas}(\partial \kappa)}{\operatorname{meas}(\kappa)}\right)^{\frac{1}{2}}\|v\|_{\kappa}, \\
& \forall v \in \mathbb{Q}_{p, d}^{0}(\kappa), \quad\|v\|_{\kappa} \leq\left(\frac{2 d}{p(p+1)}\left(2+\frac{1}{p}\right)^{d-1} \frac{\operatorname{meas}(\kappa)}{\operatorname{meas}(\partial \kappa)}\right)^{\frac{1}{2}}\|v\|_{\partial \kappa},
\end{aligned}
$$

with the convention that $\operatorname{meas}(\partial \kappa)=2$ if $d=1$.

Proof. Let $\left\{\varpi_{j}\right\}_{0 \leq j \leq p}$ be the weights associated with the one-dimensional GaußLobatto nodes. Recall that 11

$$
\varpi_{j}=\frac{2}{p(p+1)} \frac{1}{L_{p}^{2}\left(g_{j}\right)}, \quad j \in\{0, \ldots, p\},
$$

where $L_{p}$ is the Legendre polynomial of degree $p$. For a multi-index $(i)=\left(i_{1}, \ldots, i_{d}\right)$ in $I_{p, d}$, set $\varpi_{(i)}=\prod_{l=1}^{d} \varpi_{i_{l}}$. Recall that (see, e.g., [24])

$$
\forall v \in \mathbb{Q}_{p, d}(\widehat{\kappa}), \quad\|v\|_{\widehat{\kappa}} \leq\left(\sum_{(i) \in I_{p, d}} \varpi_{(i)} v\left(a_{\widehat{\kappa},(i)}\right)^{2}\right)^{\frac{1}{2}} \leq\left(2+\frac{1}{p}\right)^{\frac{d}{2}}\|v\|_{\widehat{\kappa}} .
$$

(A) Proof of (10). Let $\widehat{v} \in \mathbb{Q}_{p, d}(\widehat{\kappa})$. Set $\widehat{F}_{ \pm}=\left\{x_{1}= \pm 1\right\}$. Using the first inequality in (13) in dimension $(d-1)$ on $\widehat{F}_{ \pm}$yields

$$
\|\widehat{v}\|_{\widehat{F}_{+} \cup \widehat{F}_{-}}^{2} \leq \sum_{\left(i^{\prime}\right) \in I_{p, d-1}} \varpi_{\left(i^{\prime}\right)} \widehat{v}\left(a_{\widehat{F}_{+},\left(i^{\prime}\right)}\right)^{2}+\sum_{\left(i^{\prime}\right) \in I_{p, d-1}} \varpi_{\left(i^{\prime}\right)} \widehat{v}\left(a_{\widehat{F}_{-},\left(i^{\prime}\right)}\right)^{2},
$$


where $a_{\widehat{F}_{ \pm},\left(i^{\prime}\right)}$ are the tensor-product Gauß-Lobatto nodes on $\widehat{F}_{ \pm}$. Using the fact that $\varpi_{0}=\varpi_{p}=\frac{2}{p(p+1)}$ leads to

$$
\|\widehat{v}\|_{\widehat{F}_{+} \cup \widehat{F}_{-}}^{2} \leq \frac{p(p+1)}{2} \sum_{\substack{(i) \in I_{p, d} \\ i_{1} \in\{0, p\}}} \varpi_{(i)} \widehat{v}\left(a_{\widehat{\kappa},(i)}\right)^{2} .
$$

Hence, owing to the second inequality in (13) in dimension $d$, it is inferred that

$$
\|\widehat{v}\|_{\widehat{F}_{+} \cup \widehat{F}_{-}}^{2} \leq \frac{p(p+1)}{2} \sum_{(i) \in I_{p, d}} \varpi_{(i)} \widehat{v}\left(a_{\widehat{\kappa},(i)}\right)^{2} \leq \frac{p(p+1)}{2}\left(2+\frac{1}{p}\right)^{d}\|v\|_{\widehat{\kappa}}^{2} .
$$

Summing over all pairs of opposite faces yields

$$
\|v\|_{\partial \widehat{\kappa}}^{2} \leq d \frac{p(p+1)}{2}\left(2+\frac{1}{p}\right)^{d}\|v\|_{\widehat{\kappa}}^{2} .
$$

Let $v \in \mathbb{Q}_{p, d}(\kappa)$. Then, $v \circ T_{\kappa} \in \mathbb{Q}_{p, d}(\widehat{\kappa})$. Use (14), the fact that meas $(\widehat{\kappa})=$ $\frac{1}{d}$ meas $(\partial \widehat{\kappa})$, and a scaling argument to infer (10).

(B) Proof of (11). Let $\widehat{v} \in \mathbb{Q}_{p, d}^{0}(\widehat{\kappa})$. Using the first inequality in (13) in dimension $d$ and the second inequality in (13) in dimension $(d-1)$ yields

$$
\begin{aligned}
\|\widehat{v}\|_{\widehat{\kappa}}^{2} & \leq \sum_{(i) \in I_{p, d}} \varpi_{(i)} \widehat{v}\left(a_{\widehat{\kappa},(i)}\right)^{2}=\sum_{l=1}^{d} \sum_{\substack{(i) \in I_{p, d} \\
i_{l} \in\{0, p\}}} \varpi_{(i)} \widehat{v}\left(a_{\widehat{\kappa},(i)}\right)^{2} \\
& =\sum_{l=1}^{d} \frac{2}{p(p+1)} \sum_{\substack{(i) \in I_{p, d} \\
i_{l} \in\{0, p\}}}\left(\varpi_{i_{1}} \ldots \varpi_{i_{l-1}} \varpi_{i_{l+1}} \ldots \varpi_{i_{p}}\right) \widehat{v}\left(a_{\widehat{\kappa},(i)}\right)^{2} \\
& \leq \sum_{l=1}^{d} \frac{2}{p(p+1)}\left(2+\frac{1}{p}\right)^{d-1}\|\widehat{v}\|_{\left\{x_{l}= \pm 1\right\}}^{2}=\frac{2}{p(p+1)}\left(2+\frac{1}{p}\right)^{d-1}\|\widehat{v}\|_{\partial \widehat{\kappa}}^{2} .
\end{aligned}
$$

Let $v \in \mathbb{Q}_{p, d}^{0}(\kappa)$. Then, $v \circ T_{\kappa} \in \mathbb{Q}_{p, d}^{0}(\widehat{\kappa})$. Conclude by proceeding as above.

While the trace inequalities in Lemma 3.1 are valid in arbitrary space dimension, the focus in this paper is set on the case where $d$ is fixed, e.g., $d \leq 3$. This allows us to simplify the constants in the trace inequalities as follows:

$$
\begin{aligned}
& \forall v \in \mathbb{Q}_{p, d}(\kappa), \quad\|v\|_{\partial \kappa} \leq c\left(\frac{p^{2}}{h_{\kappa}}\right)^{\frac{1}{2}}\|v\|_{\kappa}, \\
& \forall v \in \mathbb{Q}_{p, d}^{0}(\kappa), \quad\|v\|_{\kappa} \leq c\left(\frac{h_{\kappa}}{p^{2}}\right)^{\frac{1}{2}}\|v\|_{\partial \kappa} .
\end{aligned}
$$

Inequality (15) is derived in [25] for triangular and quadrangular reference elements; inequality (16) is, to the authors' knowledge, new. An important observation is that the inverse trace inequality (16) is optimal (asymptotically in $p$ ) with respect to the trace inequality (15).

3.2. Continuous $h p$-interpolation. The goal is to construct an operator $\mathcal{I}_{\mathrm{Os}}$ : $W_{h}^{p} \rightarrow V_{h}^{p}$ endowed with a local $h p$-interpolation property. 
Let $\kappa \in \mathcal{K}$. For a node $\nu$ in $\kappa$, set $\mathcal{K}_{\nu}=\left\{\kappa^{\prime} \in \mathcal{K} ; \nu \in \kappa^{\prime}\right\}$; then, for $w_{h} \in W_{h}^{p}$, define $\mathcal{I}_{\mathrm{Os}} w_{h}$ locally in $\kappa$ by the value it takes at all the tensor-product GaußLobatto nodes by setting

$$
\mathcal{I}_{\mathrm{Os}} w_{h}(\nu)=\left.\frac{1}{\operatorname{card}\left(\mathcal{K}_{\nu}\right)} \sum_{\kappa \in \mathcal{K}_{\nu}} w_{h}\right|_{\kappa}(\nu)
$$

Clearly, $\mathcal{I}_{\mathrm{Os}} w_{h} \in V_{h}^{p}$. The operator $\mathcal{I}_{\mathrm{Os}}$ is sometimes referred to as the Oswald interpolation operator; it has been considered in [5, 13, 16, 22. Another $h p$-interpolation operator for non-smooth functions generalizing that of Clément and Scott-Zhang is analyzed in 23$]$.

Lemma 3.2. There exists $c$, independent of $p$ and $h$, such that, for all $\kappa \in \mathcal{K}$, the following estimate holds:

$$
\forall w_{h} \in W_{h}^{p}, \quad\left\|w_{h}-\mathcal{I}_{\mathrm{Os}} w_{h}\right\|_{\kappa} \leq c\left(\frac{h_{\kappa}}{p^{2}}\right)^{\frac{1}{2}} \sum_{F \in \mathcal{F}(\kappa)}\left\|\left[w_{h}\right]\right\|_{F},
$$

where $\mathcal{F}(\kappa)=\{F \in \mathcal{F} ; F \cap \kappa \neq \emptyset\}$.

Proof. Let $w_{h} \in W_{h}^{p}$ and let $\kappa \in \mathcal{K}$. Set $\delta_{h}=\left.\left(w_{h}-\mathcal{I}_{\mathrm{Os}} w_{h}\right)\right|_{\kappa}$. For each tensorproduct Gauß-Lobatto node $\nu \in \kappa$, let $\varphi_{\nu}$ denote the associated nodal basis function. By construction, $\delta_{h}$ vanishes at all the nodes located in the interior of $\kappa$. Hence,

$$
\delta_{h}=\sum_{\nu \in \partial \kappa} \delta_{h}(\nu) \varphi_{\nu}
$$

(A) Define $\partial \kappa_{d-1}$ to be the set of $(d-1)$-manifolds in $\partial \kappa$ and for $m \in$ $\{d-2, \ldots, 0\}$, define $\partial \kappa_{m}$ to be the set obtained by taking the intersection of any two distinct elements in $\partial \kappa_{m+1}$. For instance, for $d=3, \partial \kappa_{2}, \partial \kappa_{1}$, and $\partial \kappa_{0}$ are, respectively, the set of faces, edges, and vertices of $\partial \kappa$. Partition the boundary nodes of $\kappa$ as follows. For $m \in\{0, \ldots, d-1\}$, denote by $\mathcal{V}_{m}$ the set of nodes in the interior of $\partial \kappa_{m}$; then, $\bigcup_{m=0}^{d-1} \mathcal{V}_{m}$ forms a partition of the boundary nodes of $\kappa$. Hence,

$$
\delta_{h}=\sum_{\nu \in \mathcal{V}_{d-1}} \delta_{h}(\nu) \varphi_{\nu}+\sum_{m=0}^{d-2} r_{m}
$$

with

$$
r_{m}=\sum_{\nu \in \mathcal{V}_{m}} \delta_{h}(\nu) \varphi_{\nu}
$$

Observe that for all $m \in\{0, \ldots, d-2\}, r_{m} \in \mathbb{Q}_{p, d}^{0}(\kappa)$ and

$$
r_{m} \in \mathbb{Q}_{p, l}^{0}\left(\partial \kappa_{l}\right), \quad \forall l \in\{m+1, \ldots, d-1\} .
$$

(B) On each face $F \subset \partial \kappa$ and for each node $\nu$ located in the interior of $F$, equation (17) implies that $\delta_{h}(\nu)=\epsilon_{F}\left[w_{h}\right]_{F}(\nu)$ with $\epsilon_{F}= \pm \frac{1}{2}$. Hence,

$$
\sum_{\nu \in \mathcal{V}_{d-1}} \delta_{h}(\nu) \varphi_{\nu}=\sum_{F \subset \partial \kappa} \psi_{F},
$$

with

$$
\psi_{F}=\epsilon_{F} \sum_{\substack{\circ \\ \nu}}\left[w_{h}\right]_{F}(\nu) \varphi_{\nu}
$$


where $\stackrel{\circ}{F}$ denotes the interior of $F$. Since $\psi_{F} \in \mathbb{Q}_{p, d}^{0}(\kappa)$ and $\psi_{F}$ vanishes on $\partial \kappa \backslash F$, the inverse trace inequality (16) yields

$$
\left\|\psi_{F}\right\|_{\kappa} \leq c\left(\frac{h_{\kappa}}{p^{2}}\right)^{\frac{1}{2}}\left\|\psi_{F}\right\|_{\partial \kappa}=c\left(\frac{h_{\kappa}}{p^{2}}\right)^{\frac{1}{2}}\left\|\psi_{F}\right\|_{F} .
$$

Hence,

$$
\left\|\delta_{h}\right\|_{\kappa} \leq c \sum_{F \subset \partial \kappa}\left(\frac{h_{\kappa}}{p^{2}}\right)^{\frac{1}{2}}\left\|\psi_{F}\right\|_{F}+\sum_{m=0}^{d-2}\left\|r_{m}\right\|_{\kappa}:=(\mathrm{I})+(\mathrm{II}) .
$$

The rest of the proof consists of estimating both terms in the right-hand side of (19). If $d=1$, term (II) vanishes and $\left.\psi_{F}\right|_{F}=\epsilon_{F}\left[w_{h}\right]_{F}$; hence, (19) readily yields (18).

(C) Assume $d=2$. Let us first consider term (I). Let $F \subset \partial \kappa$. Since $\psi_{F}-\epsilon_{F}\left[w_{h}\right]_{F}$ is in $\mathbb{Q}_{p, 1}^{0}(F)$, the inverse trace inequality (16) yields

$$
\left\|\psi_{F}-\epsilon_{F}\left[w_{h}\right]_{F}\right\|_{F} \leq c\left(\frac{h_{F}}{p^{2}}\right)^{\frac{1}{2}}\left\|\left[w_{h}\right]_{F}\right\|_{\partial F}
$$

since $\psi_{F}$ vanishes on $\partial F$. Owing to the trace inequality (15),

$$
\left\|\left[w_{h}\right]_{F}\right\|_{\partial F} \leq c\left(\frac{p^{2}}{h_{F}}\right)^{\frac{1}{2}}\left\|\left[w_{h}\right]_{F}\right\|_{F} .
$$

Inequalities (20) and (21) imply

$$
\left\|\psi_{F}-\epsilon_{F}\left[w_{h}\right]_{F}\right\|_{F} \leq c\left\|\left[w_{h}\right]_{F}\right\|_{F} .
$$

Using the triangle inequality yields $\left\|\psi_{F}\right\|_{F} \leq c\left\|\left[w_{h}\right]_{F}\right\|_{F}$; hence,

$$
\text { (I) } \leq c\left(\frac{h_{\kappa}}{p^{2}}\right)^{\frac{1}{2}} \sum_{F \subset \partial \kappa}\left\|\left[w_{h}\right]_{F}\right\|_{F} .
$$

Let us now consider term (II). Since $r_{0} \in \mathbb{Q}_{p, 2}^{0}(\kappa)$ and $r_{0} \in \mathbb{Q}_{p, 1}^{0}(F)$ for all $F \subset \partial \kappa$, using the inverse trace inequality (16) twice, yields

$$
\left\|r_{0}\right\|_{\kappa} \leq c\left(\frac{h_{\kappa}}{p^{2}}\right)^{\frac{1}{2}} \sum_{F \subset \partial \kappa}\left\|r_{0}\right\|_{F} \leq c\left(\frac{h_{\kappa}}{p^{2}}\right)^{\frac{1}{2}} \sum_{F \subset \partial \kappa}\left(\frac{h_{F}}{p^{2}}\right)^{\frac{1}{2}}\left\|r_{0}\right\|_{\partial F} .
$$

Observe that $\partial F$ consists of two nodes and that for $\nu \in \partial F$,

$$
r_{0}(\nu)=\sum_{F^{\prime} \in \mathcal{F}_{\nu}} \eta_{F, F^{\prime}}\left[w_{h}\right]_{F^{\prime}}(\nu),
$$

where $\mathcal{F}_{\nu}$ denotes the set of faces in the mesh containing the node $\nu$ and where $\eta_{F, F^{\prime}}= \pm \frac{3}{8}$ if $F^{\prime} \subset \partial \kappa$ and $\eta_{F, F^{\prime}}= \pm \frac{1}{8}$ otherwise. Then, for all $F \subset \partial \kappa$, the following holds:

$$
\begin{aligned}
\left\|r_{0}\right\|_{\partial F} & \leq c \sum_{\nu \in \partial F} \sum_{F^{\prime} \in \mathcal{F}_{\nu}}\left|\left[w_{h}\right]_{F^{\prime}}(\nu)\right| \\
& \leq c \sum_{\nu \in \partial F} \sum_{F^{\prime} \in \mathcal{F}_{\nu}}\left(\frac{p^{2}}{h_{F^{\prime}}}\right)^{\frac{1}{2}}\left\|\left[w_{h}\right]_{F^{\prime}}\right\|_{F^{\prime}} \\
& \leq c \sum_{F^{\prime} ; F \cap F^{\prime} \neq \emptyset}\left(\frac{p^{2}}{h_{F^{\prime}}}\right)^{\frac{1}{2}}\left\|\left[w_{h}\right]_{F^{\prime}}\right\|_{F^{\prime}},
\end{aligned}
$$


owing to the trace inequality (15). Hence,

$$
\begin{aligned}
(\mathrm{II}) & \leq c\left(\frac{h_{\kappa}}{p^{2}}\right)^{\frac{1}{2}} \sum_{F \subset \partial \kappa}\left(\frac{h_{F}}{p^{2}}\right)^{\frac{1}{2}} \sum_{F^{\prime} ; F \cap F^{\prime} \neq \emptyset}\left(\frac{p^{2}}{h_{F^{\prime}}}\right)^{\frac{1}{2}}\left\|\left[w_{h}\right]_{F^{\prime}}\right\|_{F^{\prime}} \\
& \leq c\left(\frac{h_{\kappa}}{p^{2}}\right)^{\frac{1}{2}} \sum_{F \in \mathcal{F}(\kappa)}\left\|\left[w_{h}\right]_{F}\right\|_{F} .
\end{aligned}
$$

The proof is complete.

(D) For $d=3$, the proof proceeds similarly. Term (I) is estimated as for $d=2$. Term (II) is equal to $\left\|r_{0}\right\|_{\kappa}+\left\|r_{1}\right\|_{\kappa}$. The quantity $\left\|r_{0}\right\|_{\kappa}$ is estimated as for $d=2$ by using the inverse trace inequality (16) three times and the trace inequality (15) twice. To estimate $\left\|r_{1}\right\|_{\kappa}$, use twice the inverse trace inequality (16) to infer

$$
\left\|r_{1}\right\|_{\kappa} \leq c\left(\frac{h_{\kappa}}{p^{2}}\right)^{\frac{1}{2}} \sum_{F \subset \partial \kappa}\left(\frac{h_{F}}{p^{2}}\right)^{\frac{1}{2}} \sum_{E \subset \partial F}\left\|r_{1}\right\|_{E},
$$

where $E \subset \partial F$ means for all edges in $\partial F$. Straightforward algebra shows that

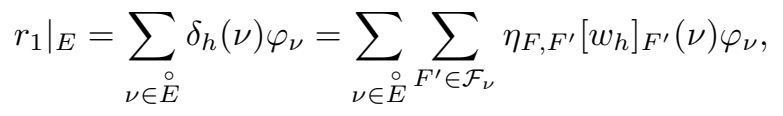

where $\stackrel{\circ}{E}$ denotes the interior of $E$. Observe that the coefficient $\eta_{F, F^{\prime}}$ is independent of $\nu$. Let $\mathcal{F}_{E}$ be the set of faces in the mesh that contain $E$. Then,

$$
\left.r_{1}\right|_{E}=\sum_{F^{\prime} \in \mathcal{F}_{E}} \eta_{F, F^{\prime}} \sum_{\substack{\mathcal{O} \in E \\ \nu \in{ }^{\prime}}}\left[w_{h}\right]_{F^{\prime}}(\nu) \varphi_{\nu}
$$

Hence,

$$
\left\|r_{1}\right\|_{E} \leq c \sum_{F^{\prime} \in \mathcal{F}_{E}}\left\|\sum_{\nu \in \mathcal{O}}\left[w_{h}\right]_{F^{\prime}}(\nu) \varphi_{\nu}\right\|_{E} \leq c \sum_{F^{\prime} \in \mathcal{F}_{E}}\left(\left\|\left[w_{h}\right]_{F^{\prime}}\right\|_{E}+\left\|\zeta_{h, F^{\prime}}\right\|_{E}\right),
$$

with $\zeta_{h, F^{\prime}}=\sum_{\nu \in E}\left[w_{h}\right]_{F^{\prime}}(\nu) \varphi_{\nu}-\left[w_{h}\right]_{F^{\prime}}$. Since $\zeta_{h, F^{\prime}} \in \mathbb{Q}_{p, 1}^{0}(E)$, the inverse trace inequality (16) yields

$$
\left\|\zeta_{h, F^{\prime}}\right\|_{E} \leq c\left(\frac{h_{E}}{p^{2}}\right)^{\frac{1}{2}} \sum_{\nu \in \partial E}\left|\left[w_{h}\right](\nu)\right| .
$$

The conclusion is now straightforward.

Remark 3.1. The conclusion of Lemma 3.2 holds in arbitrary space dimension. This can be proven by proceeding as above for $d=3$; the inverse trace inequality (16) is used $d$ times and the trace inequality (15) is used $(d-1)$ times.

Remark 3.2. $h p$-interpolation of discontinuous functions by continuous functions has been analyzed recently in the $H^{1}$-seminorm in two space dimensions on triangular and quadrangular meshes [17]. The $H^{1}$-seminorm estimate on quadrangles derived in [17, namely

$$
\forall w_{h} \in W_{h}^{p}, \quad\left\|\nabla\left(w_{h}-\mathcal{I}_{\mathrm{Os}} w_{h}\right)\right\|_{\kappa} \leq c\left(\frac{p^{2}}{h_{\kappa}}\right)^{\frac{1}{2}} \sum_{F \in \mathcal{F}(\kappa)}\left\|\left[w_{h}\right]\right\|_{F},
$$


can be inferred from Lemma 3.2 above and the well-known inverse inequality

$$
\left\|\nabla v_{h}\right\|_{\kappa} \leq c \frac{p^{2}}{h_{\kappa}}\left\|v_{h}\right\|_{\kappa}
$$

valid for all $v_{h} \in W_{h}^{p}$ (see, e.g., [10] for the proof of the $p$ version of this inequality).

3.3. $h p$-error estimate for continuous $L^{2}(\Omega)$-orthogonal projection. The purpose of this section is to investigate the approximation properties in the $L^{2}$ - and the $H^{1}$-norms of the operator $\Pi_{h}: L^{2}(\Omega) \rightarrow V_{h}^{p}$ defined as the $L^{2}(\Omega)$-orthogonal projector onto $V_{h}^{p}$.

First, we recall the following local $h p$-approximation property [10, 19]. Let $\Pi_{h}^{*}: L^{2}(\Omega) \rightarrow W_{h}^{p}$ be the $L^{2}(\Omega)$-orthogonal projector onto $W_{h}^{p}$. Then, there is $c$, independent of $p$ and $h$, such that for all $\kappa \in \mathcal{K}$ and all $w \in H^{q}(\mathcal{K}), q \geq 1$,

$$
\begin{gathered}
\left\|w-\Pi_{h}^{*} w\right\|_{\kappa} \leq c\left(\frac{h}{p}\right)^{s}\|w\|_{s, \kappa}, \\
\left\|\nabla\left(w-\Pi_{h}^{*} w\right)\right\|_{\kappa} \leq c p^{\frac{1}{2}}\left(\frac{h}{p}\right)^{s-1}\|w\|_{s, \kappa},
\end{gathered}
$$

with $s=\min (p+1, q)$. We now establish the global counterpart of (24)-(25) for the continuous $L^{2}(\Omega)$-orthogonal projector $\Pi_{h}$.

Lemma 3.3. There exists $c$, independent of $p$ and $h$, such that for all $w \in H^{q}(\Omega)$, $q \geq 1$,

$$
\begin{gathered}
\left\|w-\Pi_{h} w\right\|_{\Omega} \leq c\left(\frac{h}{p}\right)^{s}\|w\|_{s, \Omega}, \\
\left\|\nabla\left(w-\Pi_{h} w\right)\right\|_{\Omega} \leq c p^{\frac{1}{2}}\left(\frac{h}{p}\right)^{s-1}\|w\|_{s, \Omega},
\end{gathered}
$$

with $s=\min (p+1, q)$.

Proof. Let $w \in H^{q}(\Omega), q \geq 1$.

(A) Proof of (26). Since $V_{h}^{p} \subset W_{h}^{p}$, the definition of $\Pi_{h}$ and $\Pi_{h}^{*}$ leads to

$$
\left(\Pi_{h} w-\Pi_{h}^{*} w, v_{h}\right)=0, \quad \forall v_{h} \in V_{h}^{p} .
$$

Hence, $\Pi_{h} \Pi_{h}^{*} w=\Pi_{h} w$. Using the fact that $\mathcal{I}_{\mathrm{Os}}\left(\Pi_{h}^{*} w\right) \in V_{h}^{p}$ yields

$$
\left\|\Pi_{h} w-\Pi_{h}^{*} w\right\|_{\Omega}=\left\|\Pi_{h}\left(\Pi_{h}^{*} w\right)-\Pi_{h}^{*} w\right\|_{\Omega} \leq\left\|\mathcal{I}_{\mathrm{Os}}\left(\Pi_{h}^{*} w\right)-\Pi_{h}^{*} w\right\|_{\Omega} .
$$

Using Lemma 3.2, it is inferred that for all $\kappa \in \mathcal{K}$,

$$
\begin{aligned}
\left\|\mathcal{I}_{\mathrm{Os}}\left(\Pi_{h}^{*} w\right)-\Pi_{h}^{*} w\right\|_{\kappa} & \leq c\left(\frac{h_{\kappa}}{p^{2}}\right)^{\frac{1}{2}} \sum_{F \in \mathcal{F}(\kappa)}\left\|\left[\Pi_{h}^{*} w\right]_{F}\right\|_{F} \\
& \leq c\left(\frac{h_{\kappa}}{p^{2}}\right)^{\frac{1}{2}} \sum_{F \in \mathcal{F}(\kappa)}\left\|\left[\Pi_{h}^{*} w-w\right]_{F}\right\|_{F},
\end{aligned}
$$

since $[w]_{F}=0$ by assumption. Recalling the fact [19] that there is $c$, independent of $p$ and $h$, such that for all $\kappa \in \mathcal{K}$,

$$
\left\|w-\Pi_{h}^{*} w\right\|_{\partial \kappa} \leq c\left(\frac{h_{\kappa}}{p}\right)^{s-\frac{1}{2}}\|w\|_{s, \kappa}
$$


it is readily deduced that

$$
\left\|\Pi_{h} w-\Pi_{h}^{*} w\right\|_{\Omega} \leq c p^{-\frac{1}{2}}\left(\frac{h}{p}\right)^{s}\|w\|_{s, \Omega} .
$$

Conclude using the triangle inequality

$$
\left\|w-\Pi_{h} w\right\|_{\Omega} \leq\left\|w-\Pi_{h}^{*} w\right\|_{\Omega}+\left\|\Pi_{h}^{*} w-\Pi_{h} w\right\|_{\Omega},
$$

and Equation (24).

(B) Proof of (27). Let $\kappa \in \mathcal{K}$. Using the inverse inequality (23) together with (29), it is inferred that

$$
\left(\sum_{\kappa \in \mathcal{K}}\left\|\nabla\left(\Pi_{h}^{*} w-\Pi_{h} w\right)\right\|_{\kappa}^{2}\right)^{\frac{1}{2}} \leq c p^{\frac{1}{2}}\left(\frac{h}{p}\right)^{s-1}\|w\|_{s, \Omega} .
$$

Conclude using the triangle inequality and Equation (25).

Remark 3.3. Equation (29) shows that $\Pi_{h} w$ superconverges to $\Pi_{h}^{*} w$ by a factor of $p^{-\frac{1}{2}}$. This remarkable property allows us to compensate for the loss of one power of $p$ in the inverse inequality (23) and thus to recover a sub-optimality factor of $p^{\frac{1}{2}}$ in (27), which is exactly the same as in (25).

\section{Convergence analysis}

The purpose of this section is to show how the results established in 93 namely the $h p$-inverse trace inequality, the local $h p$-interpolation result, and the $h p$-error estimate for the continuous $L^{2}(\Omega)$-orthogonal projection, can be used to analyze the convergence of the $h p$-CIP finite element method introduced in $₫ 2$

Let $u$ solve (1) and let $u_{h}$ solve (7). Henceforth, it is assumed that the exact solution $u$ is smooth enough, i.e., $u \in H^{q}(\Omega), q>\frac{3}{2}$. Bounds on the approximation error $u-u_{h}$ are obtained in the spirit of Strang's Second Lemma by establishing consistency and boundedness properties for the discrete setting. Recall that the discrete setting satisfies the stability property stated in Lemma 2.1.

Lemma 4.1 (Consistency). Let $u \in H^{q}(\Omega), q>\frac{3}{2}$, solve (11) and let $u_{h}$ solve (7). Then, for all $v_{h} \in V_{h}^{p}$,

$$
a\left(u-u_{h}, v_{h}\right)+j\left(u-u_{h}, v_{h}\right)=0 .
$$

Proof. Since $u \in H^{q}(\Omega), q>\frac{3}{2}$, it is inferred that $[\nabla u \cdot n]_{F}=0$ for all $F \in \mathcal{F}$. Hence, $j\left(u, v_{h}\right)=0$ for all $v_{h} \in V_{h}^{p}$, whence (31) is readily deduced.

For all $\kappa \in \mathcal{K}$, set $\beta_{\kappa, \infty}:=\|\beta\|_{L^{\infty}\left(\Delta_{\kappa}\right)}$ where $\Delta_{\kappa}=\left\{\kappa^{\prime} \in \mathcal{K} ; \kappa \cap \kappa^{\prime} \neq \emptyset\right\}$. Introduce the (semi-)norm

$$
\|v\|_{h, \frac{1}{2}}=\left(\sum_{\kappa \in \mathcal{K}} h_{\kappa}^{-1} \beta_{\kappa, \infty}\|v\|_{\kappa}^{2}\right)^{\frac{1}{2}} .
$$

Let $\left(V_{h}^{p}\right)^{\perp}=\left\{z \in L^{2}(\Omega) ;\left(z, v_{h}\right)_{\Omega}=0, \forall v_{h} \in V_{h}^{p}\right\}$.

Lemma 4.2 (Boundedness). There is $c$, independent of $p$ and $h$, such that for all $z \in H^{q}(\mathcal{K}) \cap\left(V_{h}^{p}\right)^{\perp}, q>\frac{3}{2}$,

$$
\sup _{v_{h} \in V_{h}^{p}} \frac{a\left(z, v_{h}\right)+j\left(z, v_{h}\right)}{\left\|v_{h}\right\|_{a, j}} \leq\|z\|_{a, j}+c\left(p^{2} h^{\frac{1}{2}}+p^{\frac{\alpha}{2}-1}\right)\|z\|_{h, \frac{1}{2}} .
$$


Proof. The only term to estimate is $\left(z, \beta \cdot \nabla v_{h}\right)_{\Omega}$. Let $\beta_{h}$ be the $L^{2}(\Omega)$-orthogonal projection of $\beta$ onto $W_{h}^{0}$. Set $w_{h}=\beta_{h} \cdot \nabla v_{h}$. Observe that

$$
\left(z, \beta \cdot \nabla v_{h}\right)_{\Omega}=\left(z,\left(\beta-\beta_{h}\right) \cdot \nabla v_{h}\right)_{\Omega}+\left(z, w_{h}\right)_{\Omega} .
$$

(A) Since $\beta \in\left[W^{1, \infty}(\Omega)\right]^{d}$, the first term in the right-hand side of (34) is estimated as follows:

$$
\begin{aligned}
\left(z,\left(\beta-\beta_{h}\right) \cdot \nabla v_{h}\right)_{\Omega} & \leq\left(\sum_{\kappa \in \mathcal{K}} h_{\kappa}^{-1} \beta_{\kappa, \infty}\|z\|_{\kappa}^{2}\right)^{\frac{1}{2}}\left(\sum_{\kappa \in \mathcal{K}} h_{\kappa}^{3} \beta_{\kappa, \infty}^{-1}\|\beta\|_{W^{1, \infty}(\kappa)}^{2}\left\|\nabla v_{h}\right\|_{\kappa}^{2}\right)^{\frac{1}{2}} \\
& \leq c p^{2} h^{\frac{1}{2}}\|z\|_{h, \frac{1}{2}}\left\|v_{h}\right\|_{\Omega},
\end{aligned}
$$

owing to the inverse inequality (23).

(B) Let us estimate the second term in the right-hand side of (34). Using the fact that $z \in\left(V_{h}^{p}\right)^{\perp}$ yields

$$
\begin{aligned}
\left(z, w_{h}\right)_{\Omega} & =\left(z, w_{h}-\mathcal{I}_{\mathrm{Os}} w_{h}\right)_{\Omega} \\
& \leq\left(\sum_{\kappa \in \mathcal{K}} h_{\kappa}^{-1} \beta_{\kappa, \infty}\|z\|_{\kappa}^{2}\right)^{\frac{1}{2}}\left(\sum_{\kappa \in \mathcal{K}} h_{\kappa} \beta_{\kappa, \infty}^{-1}\left\|w_{h}-\mathcal{I}_{\mathrm{Os}} w_{h}\right\|_{\kappa}^{2}\right)^{\frac{1}{2}} \\
& \leq c\|z\|_{h, \frac{1}{2}}\left(\sum_{\kappa \in \mathcal{K}} \sum_{F \in \mathcal{F}(\kappa)} \beta_{\kappa, \infty}^{-1}\left(\frac{h_{\kappa}}{p}\right)^{2}\left\|\left[w_{h}\right]\right\|_{F}^{2}\right)^{\frac{1}{2}}
\end{aligned}
$$

owing to Lemma 3.2 . Observe that

$$
\left\|\left[w_{h}\right]\right\|_{F}=\left\|\left[\beta_{h} \cdot \nabla v_{h}\right]\right\|_{F} \leq\left\|\left[\left(\beta-\beta_{h}\right) \cdot \nabla v_{h}\right]\right\|_{F}+\left\|\left[\beta \cdot \nabla v_{h}\right]\right\|_{F} .
$$

Using the fact that $\beta \in\left[W^{1, \infty}(\Omega)\right]^{d}$, together with inequalities (15) and (23), the first term in the right-hand side of (35) is estimated as follows:

$$
\begin{aligned}
\left\|\left[\left(\beta-\beta_{h}\right) \cdot \nabla v_{h}\right]\right\|_{F} & \leq c \sum_{\kappa \supset F} h_{\kappa}\|\beta\|_{W^{1, \infty}(\kappa)}\left\|\left.\nabla v_{h}\right|_{\kappa}\right\|_{F} \\
& \leq c \sum_{\kappa \supset F} h_{\kappa}\|\beta\|_{W^{1, \infty}(\kappa)}\left(\frac{p^{2}}{h_{\kappa}}\right)^{\frac{1}{2}}\left\|\nabla v_{h}\right\|_{\kappa} \\
& \leq c \sum_{\kappa \supset F} h_{\kappa}\|\beta\|_{W^{1, \infty}(\kappa)}\left(\frac{p^{2}}{h_{\kappa}}\right)^{\frac{1}{2}} \frac{p^{2}}{h_{\kappa}}\left\|v_{h}\right\|_{\kappa} \\
& \leq c \sum_{\kappa \supset F}\|\beta\|_{W^{1, \infty}(\kappa)} \frac{p^{3}}{h_{\kappa}^{\frac{1}{2}}}\left\|v_{h}\right\|_{\kappa},
\end{aligned}
$$

where the notation $\kappa \supset F$ means for the two elements sharing $F$. Hence,

$$
\sum_{\kappa \in \mathcal{K}} \sum_{F \in \mathcal{F}(\kappa)} \beta_{\kappa, \infty}^{-1}\left(\frac{h_{\kappa}}{p}\right)^{2}\left\|\left[\left(\beta-\beta_{h}\right) \cdot \nabla v_{h}\right]\right\|_{F}^{2} \leq c p^{4} h\left\|v_{h}\right\|_{\Omega}^{2} .
$$


Furthermore, notice that $\left|\left[\beta \cdot \nabla v_{h}\right]_{F}\right|=\left|\beta \cdot n_{F}\right|\left|\left[\nabla v_{h} \cdot n\right]_{F}\right|$ owing to the continuity of $\beta$ and $v_{h}$. Hence,

$$
\begin{aligned}
\sum_{\kappa \in \mathcal{K}} \sum_{F \in \mathcal{F}(\kappa)} \beta_{\kappa, \infty}^{-1}\left(\frac{h_{\kappa}}{p}\right)^{2}\left\|\left[\beta \cdot \nabla v_{h}\right]\right\|_{F}^{2} & \leq c \sum_{F \in \mathcal{F}}\left(\frac{h_{F}}{p}\right)^{2} \beta_{\infty, F}\left\|\left[\nabla v_{h} \cdot n\right]\right\|_{F}^{2} \\
& \leq c p^{\alpha-2} j\left(v_{h}, v_{h}\right) .
\end{aligned}
$$

Collecting the above estimates yields

$$
\left(z, w_{h}\right)_{\Omega} \leq c\|z\|_{h, \frac{1}{2}}\left(p^{2} h^{\frac{1}{2}}+p^{\frac{\alpha}{2}-1}\right)\left\|v_{h}\right\|_{a, j} .
$$

The proof is complete.

Lemma 4.3. There is $c$, independent of $p$ and $h$, such that

$$
\left\|u-u_{h}\right\|_{a, j} \leq c\left\|u-\Pi_{h} u\right\|_{a, j}+c\left(p^{2} h^{\frac{1}{2}}+p^{\frac{\alpha}{2}-1}\right)\left\|u-\Pi_{h} u\right\|_{h, \frac{1}{2}} .
$$

Proof. Owing to Lemmata 2.1 and 4.1 .

$$
\begin{aligned}
\left\|\Pi_{h} u-u_{h}\right\|_{a, j}^{2} & \leq a\left(\Pi_{h} u-u_{h}, \Pi_{h} u-u_{h}\right)+j\left(\Pi_{h} u-u_{h}, \Pi_{h} u-u_{h}\right) \\
& =a\left(\Pi_{h} u-u, \Pi_{h} u-u_{h}\right)+j\left(\Pi_{h} u-u, \Pi_{h} u-u_{h}\right) .
\end{aligned}
$$

Since $\Pi_{h} u-u \in\left(V_{h}^{p}\right)^{\perp}$, Lemma 4.2 leads to

$$
\left\|\Pi_{h} u-u_{h}\right\|_{a, j} \leq\left\|\Pi_{h} u-u\right\|_{a, j}+c\left(p^{2} h^{\frac{1}{2}}+p^{\frac{\alpha}{2}-1}\right)\left\|\Pi_{h} u-u\right\|_{h, \frac{1}{2}} .
$$

Conclude using the triangle inequality.

Lemma 4.4. There is $c$, independent of $p$ and $h$, such that for all $w \in H^{q}(\Omega)$, $q>\frac{3}{2}$

$$
\begin{aligned}
& \left\|w-\Pi_{h} w\right\|_{h, \frac{1}{2}} \leq c p^{-\frac{1}{2}}\left(\frac{h}{p}\right)^{s-\frac{1}{2}}\|w\|_{s, \Omega}, \\
& \left\|w-\Pi_{h} w\right\|_{a, j} \leq c\left(p^{\frac{1}{4}}+p^{2-\frac{\alpha}{2}}\right)\left(\frac{h}{p}\right)^{s-\frac{1}{2}}\|w\|_{s, \Omega},
\end{aligned}
$$

with $s=\min (p+1, q)$.

Proof. Estimate (37) directly results from (26). To prove (38), first notice that owing to the standard trace inequality

$$
\|v\|_{\partial \Omega} \leq c\|v\|_{\Omega}^{\frac{1}{2}}\|\nabla v\|_{\Omega}^{\frac{1}{2}}
$$

valid for all $v \in H^{1}(\Omega)$, it is inferred from Lemma 3.3 that

$$
\left\|w-\Pi_{h} w\right\|_{\partial \Omega} \leq c p^{\frac{1}{4}}\left(\frac{h}{p}\right)^{s-\frac{1}{2}}\|w\|_{s, \Omega} .
$$

To control $j\left(w-\Pi_{h} w, w-\Pi_{h} w\right)$, observe that for all $\kappa \in \mathcal{K}$,

$$
\begin{aligned}
\left\|\nabla\left(w-\Pi_{h} w\right)\right\|_{\partial \kappa} & \leq\left\|\nabla\left(w-\Pi_{h}^{*} w\right)\right\|_{\partial \kappa}+\left\|\nabla\left(\Pi_{h} w-\Pi_{h}^{*} w\right)\right\|_{\partial \kappa} \\
& \leq\left\|\nabla\left(w-\Pi_{h}^{*} w\right)\right\|_{\partial \kappa}+p h_{\kappa}^{-\frac{1}{2}}\left\|\nabla\left(\Pi_{h} w-\Pi_{h}^{*} w\right)\right\|_{\kappa},
\end{aligned}
$$

owing to the trace inequality (15). Recall the fact [19] that there is $c$, independent of $p$ and $h$, such that for all $\kappa \in \mathcal{K}$,

$$
\left\|\nabla\left(w-\Pi_{h}^{*} w\right)\right\|_{\partial \kappa} \leq c p\left(\frac{h_{\kappa}}{p}\right)^{s-\frac{3}{2}}\|w\|_{s, \kappa} .
$$


As a result,

$$
\begin{aligned}
j\left(w-\Pi_{h} w, w\right. & \left.-\Pi_{h} w\right) \leq \sum_{F \in \mathcal{F}} h_{F}^{2} p^{-\alpha} \beta_{\infty, F}\left\|\left[\nabla\left(w-\Pi_{h} w\right) \cdot n\right]\right\|_{F}^{2} \\
& \leq c \sum_{\kappa \in \mathcal{K}}\left(h_{\kappa}^{2} p^{-\alpha}\left\|\nabla\left(w-\Pi_{h}^{*} w\right)\right\|_{\partial \kappa}^{2}+h_{\kappa} p^{2-\alpha}\left\|\nabla\left(\Pi_{h} w-\Pi_{h}^{*} w\right)\right\|_{\kappa}^{2}\right) \\
& \leq c\left(p^{4-\alpha}\left(\frac{h}{p}\right)^{2 s-1}\|w\|_{s, \Omega}^{2}+h p^{2-\alpha} \sum_{\kappa \in \mathcal{K}}\left\|\nabla\left(\Pi_{h} w-\Pi_{h}^{*} w\right)\right\|_{\kappa}^{2}\right) \\
& \leq c p^{4-\alpha}\left(\frac{h}{p}\right)^{2 s-1}\|w\|_{s, \Omega}^{2},
\end{aligned}
$$

owing to (30). The conclusion is straightforward.

We are now in a position to state the main result of this section.

Theorem 4.1. Let $u \in H^{q}(\Omega), q>\frac{3}{2}$, solve (11) and let $u_{h}$ solve (7). Take

$$
\alpha=\frac{7}{2} \text {. }
$$

Then, there is $c$, independent of $p$ and $h$, such that

$$
\left\|u-u_{h}\right\|_{a, j} \leq c\left(p^{\frac{1}{4}}+p^{\frac{3}{2}} h^{\frac{1}{2}}\right)\left(\frac{h}{p}\right)^{s-\frac{1}{2}}\|u\|_{s, \Omega},
$$

with $s=\min (p+1, q)$. Hence, if $h \leq p^{-\frac{5}{2}}$, the following holds:

$$
\left\|u-u_{h}\right\|_{a, j} \leq c p^{\frac{1}{4}}\left(\frac{h}{p}\right)^{s-\frac{1}{2}}\|u\|_{s, \Omega} .
$$

Proof. Since $\frac{\alpha}{2}-1=\frac{3}{4}$, Lemma 4.3 yields

$$
\begin{aligned}
\left\|u-u_{h}\right\|_{a, j} & \leq c\left\|u-\Pi_{h} u\right\|_{a, j}+c\left(p^{2} h^{\frac{1}{2}}+p^{\frac{3}{4}}\right)\left\|u-\Pi_{h} u\right\|_{h, \frac{1}{2}} \\
& \leq c\left\|u-\Pi_{h} u\right\|_{a, j}+c\left(p^{\frac{3}{2}} h^{\frac{1}{2}}+p^{\frac{1}{4}}\right)\left(\frac{h}{p}\right)^{s-\frac{1}{2}}\|w\|_{s, \Omega},
\end{aligned}
$$

owing to (37). Since $2-\frac{\alpha}{2}=\frac{1}{4}$, (38) leads to

$$
\left\|u-\Pi_{h} u\right\|_{a, j} \leq c p^{\frac{1}{4}}\left(\frac{h}{p}\right)^{s-\frac{1}{2}}\|u\|_{s, \Omega},
$$

whence (42) is readily deduced. Finally, under the assumption that $h \leq p^{-\frac{5}{2}}$, (43) directly results from (42).

\section{EXtension to SIMPLiCES}

The goal of this section is to discuss the extension of the analysis presented in $\S \$ 2$ to simplicial finite elements. Several points differ with respect to the case of tensor-product finite elements. First, we prove that the inverse trace inequality cannot be optimal on simplices and that, at best, a factor $p^{\frac{1}{2}}$ is lost (see Lemma 5.2). This leaves open the question of an optimal $h p$-interpolation operator from discontinuous to continuous simplicial finite element spaces. Restricting ourselves to two space dimensions, we will assume that only the factor $p^{\frac{1}{2}}$ is lost in the inverse trace inequality; see Hypothesis 5.1. Furthermore, the convergence analysis relies on the 
assumption that the local $h p$-approximation properties (24), (25), and (40) hold also on simplices; see Hypothesis 5.2. However, we will not assume that (28) holds.

Let $\mathcal{K}$ be a subdivision of $\Omega$ into simplices $\{\kappa\}$. Assume that (i) $\mathcal{K}$ covers $\bar{\Omega}$ exactly, (ii) $\mathcal{K}$ does not contain any hanging nodes, and (iii) $\mathcal{K}$ is locally quasiuniform in the sense that there exists a constant $\rho>0$, independent of $h$, such that $\rho \max _{E \subset \Delta_{\kappa}} h_{E} \leq \min _{E \subset \Delta_{\kappa}} h_{E}$ where $E \subset \Delta_{\kappa}$ means for all edges in the patch $\Delta_{\kappa}$ and $h_{E}$ denotes the diameter of $E$. It is assumed that each $\kappa \in \mathcal{K}$ is an affine image of the canonical $d$-dimensional simplex in $\mathbb{R}^{d}$ defined by $\widehat{\kappa}=\left\{\left(x_{1}, \ldots, x_{d}\right) \in\right.$ $\left.\mathbb{R}^{d} ;\left|x_{i}\right| \leq 1 ; \sum_{i=1}^{d} x_{i} \leq 2-d\right\}$.

Let $p \geq 1$ and let $\mathbb{P}_{p, d}(\widehat{\kappa})$ be the space of polynomials of total degree at most $p$. Introduce the finite element spaces

$$
\begin{aligned}
X_{h}^{p} & =\left\{v_{h} \in C^{0}(\bar{\Omega}) ; \forall \kappa \in \mathcal{K},\left.v_{h}\right|_{\kappa} \in \mathbb{P}_{p, d}(\kappa)\right\}, \\
Y_{h}^{p} & =\left\{w_{h} \in L^{2}(\Omega) ; \forall \kappa \in \mathcal{K},\left.w_{h}\right|_{\kappa} \in \mathbb{P}_{p, d}(\kappa)\right\} .
\end{aligned}
$$

5.1. Trace and inverse trace inequalities on simplices. The results presented in this section are stated in arbitrary space dimension since they can be of independent interest. First, recall the following trace inequality due to Warburton and Hesthaven [26].

Lemma 5.1. Let $\kappa \in \mathcal{K}$. The following holds:

$$
\forall v \in \mathbb{P}_{p, d}(\kappa), \quad\|v\|_{\partial \kappa} \leq\left(\frac{(p+1)(p+d)}{d} \frac{\operatorname{meas}(\partial \kappa)}{\operatorname{meas}(\kappa)}\right)^{\frac{1}{2}}\|v\|_{\kappa} .
$$

To investigate inverse trace inequalities in $\mathbb{P}_{p, d}(\kappa)$, set $n_{p, d}=\operatorname{dim}\left(\mathbb{P}_{p, d}\right)$ and let $\left\{\phi_{i}\right\}_{1 \leq i \leq n_{p, d}}$ be the canonical basis of $\mathbb{P}_{p, d}(\kappa)$. Let $\mathcal{A}=\left\{a_{i}\right\}_{1 \leq i \leq n_{p, d}}$ be a set of nodes in $\kappa$. The set $\mathcal{A}$ is said to be unisolvent in $\mathbb{P}_{p, d}(\kappa)$ if the matrix with coefficients $\left(\phi_{i}\left(a_{j}\right)\right)_{1 \leq i, j \leq n_{p, d}}$ is invertible. It is said to be admissible if it is unisolvent in $\mathbb{P}_{p, d}(\kappa)$ and if for all dimension $d^{\prime}<d$ and for any simplex $\kappa^{\prime} \subset \partial \kappa$ of dimension $d^{\prime}$, the set of nodes in $\kappa^{\prime}$ is unisolvent in $\mathbb{P}_{p, d^{\prime}}\left(\kappa^{\prime}\right)$. Given a unisolvent set of nodes $\mathcal{A}$, define

$$
\mathbb{P}_{p, d}^{\mathcal{A}}(\kappa)=\left\{v \in \mathbb{P}_{p, d}(\kappa) ; \forall a_{i} \in \mathcal{A}, v\left(a_{i}\right)=0 \text { if } a_{i} \notin \partial \kappa\right\} .
$$

Lemma 5.2. For all unisolvent set of nodes $\mathcal{A}$ in $\mathbb{P}_{p, d}(\kappa)$, the following holds:

$$
\inf _{v \in \mathbb{P}_{p, d}^{\mathcal{A}}(\kappa) \backslash\{0\}} \frac{\|v\|_{\partial \kappa}}{\|v\|_{\kappa}} \leq\left(\frac{2 p+d}{d} \frac{\operatorname{meas}(\partial \kappa)}{\operatorname{meas}(\kappa)}\right)^{\frac{1}{2}} .
$$

Proof. Estimate (48) is established on the reference simplex and then mapped to an arbitrary element in $\mathcal{K}$.

(A) Choose the $L^{2}(\widehat{\kappa})$-orthonormal basis of $\mathbb{P}_{p, d}(\widehat{\kappa})$ considered in 26. Define the matrix $\widehat{F}$ of order $n_{p, d}$ such that $(\widehat{V}, \widehat{F} \widehat{V})_{\mathbb{R}^{n_{p, d}}}=\|\widehat{v}\|_{\partial \widehat{\kappa}}^{2}$ for all $\widehat{v} \in \mathbb{P}_{p, d}(\widehat{\kappa})$, where $\widehat{V}$ is the coordinate vector of $\widehat{v}$ in the selected basis and where $(\cdot, \cdot)_{\mathbb{R}^{n}{ }_{p, d}}$ denotes the Euclidean scalar product in $\mathbb{R}^{n_{p, d}}$. Clearly, $\widehat{F}$ is symmetric positive semidefinite. The advantage of the basis selected in $[26$ is that $\widehat{F}$ admits a block-structure with blocks having rank 1.

(B) To specify this structure, define $J_{p, d}=\left\{(i) \in I_{p, d}, \sum_{l=1}^{d} i_{l} \leq p\right\}$. The matrix $\widehat{F}$ is then conveniently indexed by multi-indices $(i)$ and $(j)$ in $J_{p, d}$, and its entries 
are given by

$$
\widehat{F}_{(i)(j)}=\prod_{l=1}^{d-1} \delta_{i_{l} j_{l}}(-1)^{i_{d}}\left(\frac{2 N_{d}(i)+d}{2}\right)^{\frac{1}{2}}(-1)^{j_{d}}\left(\frac{2 N_{d}(j)+d}{2}\right)^{\frac{1}{2}},
$$

where $N_{d}(i)=\sum_{l=1}^{d} i_{l}$. Let $r \in\{0, \ldots, p\}$. With any $\left(i^{\prime}\right) \in I_{p, d-1}$ such that $\sum_{l=1}^{d-1} i_{l}^{\prime}=r$, we can associate a block $\widehat{F}_{r,\left(i^{\prime}\right)}$ in $\widehat{F}$ corresponding to the multiindices $(i)=\left(i_{1}^{\prime}, \ldots, i_{d-1}^{\prime}, i_{d}\right)$. In [26, it is proven that the block $\widehat{F}_{r,\left(i^{\prime}\right)}$ is of size $(p-r+1)$, of rank 1 , and its trace is equal to

$$
\operatorname{tr}\left(\widehat{F}_{r,\left(i^{\prime}\right)}\right)=\sum_{i_{d}=0}^{p-r} \frac{1}{2}\left(2 r+2 i_{d}+d\right)=\frac{1}{2}(p-r+1)(p+r+d) .
$$

(C) In [26, the largest eigenvalue of $\widehat{F}$ is estimated whereas here we are interested in the lowest non-zero eigenvalue. Since the blocks $\widehat{F}_{r,\left(i^{\prime}\right)}$ are of rank 1, the spectrum of $\widehat{F}$ is equal to

$$
\sigma(\widehat{F})=\left\{\frac{1}{2}(p-r+1)(p+r+d)\right\}_{0 \leq r \leq p} \cup\{0\} .
$$

Since the product $(p-r+1)(p+r+d)$ is monotonically decreasing in $r$, it is inferred that the smallest non-zero eigenvalue of $\widehat{F}$ is $\frac{2 p+d}{2}$. The conclusion is straightforward.

The result of Lemma 5.2 which is to the authors' knowledge new, has important consequences for the $h p$-convergence analysis on simplices since it implies that in space dimension $d \geq 2$, the inverse trace inequality on simplices cannot be optimal with respect to the trace inequality and that, at best, a factor $p^{\frac{1}{2}}$ is lost. In other words, for the inverse trace inequality to be optimal, one should prove that

$$
\inf _{v \in \mathbb{P}_{p, d}^{\mathcal{A}}(\kappa) \backslash\{0\}} \frac{\|v\|_{\partial \kappa}}{\|v\|_{\kappa}} \geq c\left(p^{2} \frac{\operatorname{meas}(\partial \kappa)}{\operatorname{meas}(\kappa)}\right)^{\frac{1}{2}},
$$

for a constant $c$ independent of $p$. Hence, the upper bound in (48) is too small by a factor proportional to $p^{\frac{1}{2}}$, and in the best situation where (48) is sharp, the inverse trace inequality is suboptimal by a factor of $p^{\frac{1}{2}}$ only.

The rest of the analysis presented in this section is restricted to triangles $(d=2)$ and relies on the following hypothesis, which means that (48) is sharp.

Hypothesis 5.1. There exists an admissible set of nodes $\mathcal{A}^{*}$ in $\mathbb{P}_{p, 2}(\widehat{\kappa})$ such that

$$
\forall v \in \mathbb{P}_{p, 2}^{\mathcal{A}^{*}}(\widehat{\kappa}), \quad\|v\|_{\widehat{\kappa}} \leq c p^{-\frac{1}{2}}\|v\|_{\partial \widehat{\kappa}} .
$$

Henceforth, for all $\kappa \in \mathcal{K}, \mathbb{P}_{p, d}^{0}(\kappa)$ denotes the polynomial set $\mathbb{P}_{p, 2}^{\mathcal{A}_{\kappa}^{*}}(\kappa)$ where $\mathcal{A}_{\kappa}^{*}$ is the image of the set $\mathcal{A}^{*}$ by the transformation mapping $\widehat{\kappa}$ onto $\kappa$. If $d$ is not large with respect to $p$, estimate (46) and Hypothesis 5.1 lead to the following trace and inverse trace inequalities on triangles:

$$
\begin{aligned}
& \forall v \in \mathbb{P}_{p, d}(\kappa), \quad\|v\|_{\partial \kappa} \leq c\left(\frac{p^{2}}{h_{\kappa}}\right)^{\frac{1}{2}}\|v\|_{\kappa}, \\
& \forall v \in \mathbb{P}_{p, d}^{0}(\kappa), \quad\|v\|_{\kappa} \leq c\left(\frac{h_{\kappa}}{p}\right)^{\frac{1}{2}}\|v\|_{\partial \kappa} .
\end{aligned}
$$


Inequality (51) is derived in 25]. Inequality (52) is suboptimal by a factor $p^{\frac{1}{2}}$ with respect to (51). Inequality (52) should be compared with inequality (16) derived on tensor-product elements, the latter inequality being optimal with respect to the corresponding trace inequality (15).

5.2. $h p$-interpolation and projection on triangles. Let $\kappa \in \mathcal{K}$. For a node $\nu$ in $\mathcal{A}_{\kappa}^{*}$, set $\mathcal{K}_{\nu}=\left\{\kappa^{\prime} \in \mathcal{K} ; \nu \in \kappa^{\prime}\right\}$; then, for $w_{h} \in Y_{h}^{p}$, define $\mathcal{I}_{\text {Os }} w_{h}$ locally in $\kappa$ by the value it takes at all the nodes in $\mathcal{A}_{\kappa}^{*}$ by setting

$$
\mathcal{I}_{\mathrm{Os}} w_{h}(\nu)=\left.\frac{1}{\operatorname{card}\left(\mathcal{K}_{\nu}\right)} \sum_{\kappa \in \mathcal{K}_{\nu}} w_{h}\right|_{\kappa}(\nu)
$$

Clearly, $\mathcal{I}_{\mathrm{Os}} w_{h} \in X_{h}^{p}$.

Lemma 5.3. There exists $c$, independent of $p$ and $h$, such that, for all $\kappa \in \mathcal{K}$, the following estimate holds:

$$
\forall w_{h} \in Y_{h}^{p}, \quad\left\|w_{h}-\mathcal{I}_{\mathrm{Os}} w_{h}\right\|_{\kappa} \leq c\left(\frac{h_{\kappa}}{p}\right)^{\frac{1}{2}} \sum_{F \in \mathcal{F}(\kappa)}\left\|\left[w_{h}\right]\right\|_{F} .
$$

Proof. The proof is the same as that of Lemma 3.2 except that the trace and inverse trace inequalities (51) and (52) are used instead of (15) and (16). In dimension 2, the inverse trace inequality (52) is used once so that a factor of $p^{-\frac{1}{2}}$ is lost in (54) with respect to (18).

Remark 5.1. In dimension 3, the inverse trace inequality (52) has to be used twice so that a factor of $p$ is lost.

To derive $h p$-approximation results for the $L^{2}(\Omega)$-orthogonal projector onto $X_{h}^{p}$, we make henceforth the following assumption.

Hypothesis 5.2. The $L^{2}(\Omega)$-orthogonal projector onto $X_{h}^{p}$ satisfies the hp-approximation results (24), (25), and (40).

Lemma 5.4. Let $\Pi_{h}: L^{2}(\Omega) \rightarrow X_{h}^{p}$ be the $L^{2}(\Omega)$-orthogonal projector onto $X_{h}^{p}$. There exists $c$, independent of $p$ and $h$, such that for all $w \in H^{q}(\Omega), q \geq 1$,

$$
\begin{gathered}
\left\|w-\Pi_{h} w\right\|_{\Omega} \leq c p^{\frac{1}{4}}\left(\frac{h}{p}\right)^{s}\|w\|_{s, \Omega}, \\
\left\|\nabla\left(w-\Pi_{h} w\right)\right\|_{\Omega} \leq c p^{\frac{5}{4}}\left(\frac{h}{p}\right)^{s-1}\|w\|_{s, \Omega},
\end{gathered}
$$

with $s=\min (p+1, q)$.

Proof. The proof proceeds as that of Lemma 3.3 except that the bound (54) is used instead of (18) and that instead of (28), we use the inequality

$$
\left\|w-\Pi_{h}^{*} w\right\|_{\partial \kappa} \leq c p^{\frac{1}{4}}\left(\frac{h_{\kappa}}{p}\right)^{s-\frac{1}{2}}\|w\|_{s, \kappa},
$$

resulting from Hypothesis 5.2 and the standard trace inequality (39). 
5.3. Convergence analysis on triangles. Let $u \in H^{q}(\Omega), q>\frac{3}{2}$, solve (11) and let $u_{h} \in X_{h}^{p}$ solve

$$
a\left(u_{h}, v_{h}\right)+j\left(u_{h}, v_{h}\right)=\left(f, v_{h}\right)_{\Omega}, \quad \forall v_{h} \in X_{h}^{p} .
$$

The convergence analysis proceeds as in $₫ 4$. Let $\left(X_{h}^{p}\right)^{\perp}=\left\{z \in L^{2}(\Omega) ;\left(z, v_{h}\right)_{\Omega}=\right.$ $\left.0, \forall v_{h} \in X_{h}^{p}\right\}$.

Lemma 5.5 (Boundedness). There is $c$, independent of $p$ and $h$, such that for all $z \in H^{q}(\mathcal{K}) \cap\left(X_{h}^{p}\right)^{\perp}, q>\frac{3}{2}$,

$$
\sup _{v_{h} \in X_{h}^{p}} \frac{a\left(z, v_{h}\right)+j\left(z, v_{h}\right)}{\left\|v_{h}\right\|_{a, j}} \leq\|z\|_{a, j}+c\left(p^{2} h^{\frac{1}{2}}+p^{\frac{\alpha}{2}-\frac{1}{2}}\right)\|z\|_{h, \frac{1}{2}} .
$$

Proof. The proof is similar to that of Lemma 4.2. However, on simplices, $\beta_{h}$ can be chosen to be in $X_{h}^{1}$. As a result, there is no need to invoke the triangle inequality (35), and it is directly inferred using (54) that

$$
\sum_{\kappa \in \mathcal{K}} \sum_{F \in \mathcal{F}(\kappa)} \beta_{\kappa, \infty}^{-1} h_{\kappa}^{2} p^{-1}\left\|\left[\beta_{h} \cdot \nabla v_{h}\right]\right\|_{F}^{2} \leq c p^{\alpha-1} j\left(v_{h}, v_{h}\right) .
$$

The conclusion is straightforward.

Lemma 5.6. There is $c$, independent of $p$ and $h$, such that for all $w \in H^{q}(\Omega)$, $q>\frac{3}{2}$

$$
\begin{aligned}
\left\|w-\Pi_{h} w\right\|_{h, \frac{1}{2}} & \leq c p^{-\frac{1}{4}}\left(\frac{h}{p}\right)^{s-\frac{1}{2}}\|w\|_{s, \Omega}, \\
\left\|w-\Pi_{h} w\right\|_{a, j} & \leq c\left(p^{\frac{3}{4}}+p^{\frac{11}{4}-\frac{\alpha}{2}}\right)\left(\frac{h}{p}\right)^{s-\frac{1}{2}}\|w\|_{s, \Omega},
\end{aligned}
$$

with $s=\min (p+1, q)$.

Proof. Similar to that of Lemma 4.4.

We are now in a position to state the main result of this section.

Theorem 5.1. Let $u \in H^{q}(\Omega), q>\frac{3}{2}$, solve (11) and let $u_{h}$ solve (57). Take $\alpha=\frac{7}{2}$. Then, under Hypotheses 5.1 and [5.2, there is $c$, independent of $p$ and $h$, such that

$$
\left\|u-u_{h}\right\|_{a, j} \leq c\left(p+p^{\frac{7}{4}} h^{\frac{1}{2}}\right)\left(\frac{h}{p}\right)^{s-\frac{1}{2}}\|u\|_{s, \Omega},
$$

with $s=\min (p+1, q)$. Hence, if $h \leq p^{-\frac{3}{2}}$, the following holds:

$$
\left\|u-u_{h}\right\|_{a, j} \leq c p\left(\frac{h}{p}\right)^{s-\frac{1}{2}}\|u\|_{s, \Omega} .
$$

Proof. Proceeding as in the proof of Theorem 4.1, it is inferred that

$$
\left\|u-u_{h}\right\|_{a, j} \leq c\left(p^{\frac{3}{4}}+p^{\frac{11}{4}-\frac{\alpha}{2}}+p^{\frac{\alpha}{2}-\frac{3}{4}}+p^{\frac{7}{4}} h^{\frac{1}{2}}\right)\left(\frac{h}{p}\right)^{s-\frac{1}{2}}\|u\|_{s, \Omega} .
$$

Since $\alpha=\frac{7}{2}$, it is clear that $\frac{11}{4}-\frac{\alpha}{2}=\frac{\alpha}{2}-\frac{3}{4}=1 \geq \frac{3}{4}$. The conclusion is straightforward. 


\section{Advection-Diffusion problems}

The purpose of this section is to show that the $h p$-CIP bilinear form $j$ defined by equation (6) is also suitable to approximate advection-diffusion problems in both the advection-dominated and the diffusion-dominated regimes. The discussion is restricted to finite element approximations on tensor-product meshes.

Consider the following PDE with mixed Neumann-Robin boundary conditions

$$
\left\{\begin{aligned}
-\varepsilon \Delta u+\beta \cdot \nabla u+\sigma u & =f, \\
\left.(\beta u-\varepsilon \nabla u) \cdot n\right|_{\partial \Omega^{-}} & =0, \\
\left.\varepsilon \nabla u \cdot n\right|_{\partial \Omega \backslash \partial \Omega^{-}} & =0,
\end{aligned}\right.
$$

where $\varepsilon>0$ is a real parameter and where $f, \beta$, and $\sigma$ satisfy the assumptions stated in $\S 2$ Without loss of generality, it is assumed that problem (63) is nondimensionalized so that $\beta$ is of order unity. Note that Neumann and Robin boundary conditions are natural outflow and inflow conditions, respectively, for advectiondiffusion problems.

On $H^{1}(\Omega) \times H^{1}(\Omega)$ define the standard Galerkin bilinear form

$$
a_{\varepsilon}(u, v)=\varepsilon(\nabla u, \nabla v)_{\Omega}+((\sigma-\nabla \cdot \beta) u, v)_{\Omega}-(u, \beta \cdot \nabla v)_{\Omega}+(\beta \cdot n u, v)_{\partial \Omega^{+}} .
$$

The finite element approximation to (63) consists of finding $u_{h} \in V_{h}^{p}$ such that

$$
a_{\varepsilon}\left(u_{h}, v_{h}\right)+j\left(u_{h}, v_{h}\right)=\left(f, v_{h}\right)_{\Omega}, \quad \forall v_{h} \in V_{h}^{p} .
$$

For $v \in H^{q}(\mathcal{K}), q>\frac{3}{2}$, consider the norm

$$
\|v\|_{\varepsilon, j}^{2}=\left\|\varepsilon^{\frac{1}{2}} \nabla v\right\|_{\Omega}^{2}+\left\|\sigma_{0}^{\frac{1}{2}} v\right\|_{\Omega}^{2}+\frac{1}{2}\left\||\beta \cdot n|^{\frac{1}{2}} v\right\|_{\partial \Omega}^{2}+j(v, v) .
$$

The discrete problem (65) is clearly endowed with stability and consistency properties.

Lemma 6.1 (Coerciveness). For all $v \in H^{q}(\mathcal{K}), q>\frac{3}{2}, a_{\varepsilon}(v, v)+j(v, v) \geq\|v\|_{\varepsilon, j}^{2}$.

Lemma 6.2 (Consistency). Let $u \in H^{q}(\Omega), q>\frac{3}{2}$, solve (63) and let $u_{h}$ solve (65). Then, for all $v_{h} \in V_{h}^{p}$,

$$
a_{\varepsilon}\left(u-u_{h}, v_{h}\right)+j\left(u-u_{h}, v_{h}\right)=0 .
$$

Proposition 6.1 (Dominant advection). Let $u \in H^{q}(\Omega), q>\frac{3}{2}$, solve (63) and let $u_{h}$ solve (65). Take $\alpha=\frac{7}{2}$. Assume $h \leq p^{-\frac{5}{2}}$. Then, there is $c$, independent of $p$, $h$, and $\epsilon$, such that

$$
\left\|u-u_{h}\right\|_{\varepsilon, j} \leq c\left(\varepsilon^{\frac{1}{2}} p h^{-\frac{1}{2}}+p^{\frac{1}{4}}\right)\left(\frac{h}{p}\right)^{s-\frac{1}{2}}\|u\|_{s, \Omega},
$$

with $s=\min (p+1, q)$. Hence, if $\varepsilon \leq h p^{-\frac{3}{2}}$, the following holds:

$$
\left\|u-u_{h}\right\|_{\varepsilon, j} \leq c p^{\frac{1}{4}}\left(\frac{h}{p}\right)^{s-\frac{1}{2}}\|u\|_{s, \Omega} .
$$

Proof. Proceed as in the proof of Theorem 4.1. The only additional term to estimate is $\left\|\varepsilon^{\frac{1}{2}} \nabla\left(u-\Pi_{h} u\right)\right\|_{\Omega}$. Use (27) to infer

$$
\left\|\varepsilon^{\frac{1}{2}} \nabla\left(u-\Pi_{h} u\right)\right\|_{\Omega} \leq c p^{\frac{1}{2}} \varepsilon^{\frac{1}{2}}\left(\frac{h}{p}\right)^{s-1}\|u\|_{s, \Omega} .
$$

The conclusion is straightforward. 
Proposition 6.2 (Dominant diffusion). Let $u \in H^{q}(\Omega), q \geq 2$, solve (63) and let $u_{h}$ solve (65). Take $\alpha=\frac{7}{2}$. Then, there is $c$, independent of $p, h$, and $\epsilon$, such that

$$
\left\|u-u_{h}\right\|_{\varepsilon, j} \leq c\left(1+\varepsilon^{-\frac{1}{2}}\right)\left(\varepsilon^{\frac{1}{2}}+1+h^{\frac{1}{2}} p^{-\frac{3}{4}}\right)\left(\frac{h}{p}\right)^{s-1}\|u\|_{s, \Omega},
$$

with $s=\min (p+1, q)$. Hence, if $\varepsilon \geq \max \left(1, h p^{-\frac{3}{2}}\right)$, the following holds:

$$
\left\|u-u_{h}\right\|_{\varepsilon, j} \leq c \varepsilon^{\frac{1}{2}}\left(\frac{h}{p}\right)^{s-1}\|u\|_{s, \Omega} .
$$

Proof. Recall the following interpolation result [2]: there exists $u_{h}^{*} \in V_{h}^{p}$ such that

$$
\left\|u-u_{h}^{*}\right\|_{1, \Omega} \leq c\left(\frac{h}{p}\right)^{s-1}\|u\|_{s, \Omega} .
$$

(A) Let us first prove that

$$
j\left(u-u_{h}^{*}, u-u_{h}^{*}\right)^{\frac{1}{2}} \leq \operatorname{ch}^{\frac{1}{2}} p^{-\frac{3}{4}}\left(\frac{h}{p}\right)^{s-1}\|u\|_{s, \Omega} .
$$

Let $\Pi_{h, 2}^{*}: H^{2}(\Omega) \rightarrow W_{h}^{p}$ be the $H^{2}$-orthogonal projection onto $W_{h}^{p}$. Recall that $\Pi_{h, 2}^{*}$ is endowed with optimal $h p$-approximation properties in the $L^{2}$-, $H^{1}$ - and $H^{2}$-norms; see [10]. Let $\kappa \in \mathcal{K}$ and let $F \subset \partial \kappa$. Owing to the trace inequality (15), it is inferred that

$$
\begin{aligned}
\left\|\left.\nabla\left(u-u_{h}^{*}\right)\right|_{\kappa}\right\|_{F} & \leq\left\|\left.\nabla\left(u-\Pi_{h, 2}^{*} u\right)\right|_{\kappa}\right\|_{F}+\left\|\left.\nabla\left(\Pi_{h, 2}^{*} u-u_{h}^{*}\right)\right|_{\kappa}\right\|_{F} \\
& \leq c\left(\frac{h_{\kappa}}{p}\right)^{s-\frac{3}{2}}\|u\|_{s, \kappa}+c p h_{\kappa}^{-\frac{1}{2}}\left\|\nabla\left(\Pi_{h, 2}^{*} u-u_{h}^{*}\right)\right\|_{\kappa} \\
& \leq c\left(\frac{h_{\kappa}}{p}\right)^{s-\frac{3}{2}}\|u\|_{s, \kappa}+c p h_{\kappa}^{-\frac{1}{2}}\left(\left\|\nabla\left(u-\Pi_{h, 2}^{*} u\right)\right\|_{\kappa}+\left\|\nabla\left(u-u_{h}^{*}\right)\right\|_{\kappa}\right) \\
& \leq c p^{\frac{1}{2}}\left(\frac{h_{\kappa}}{p}\right)^{s-\frac{3}{2}}\|u\|_{s, \kappa}+c p h_{\kappa}^{-\frac{1}{2}}\left\|\nabla\left(u-u_{h}^{*}\right)\right\|_{\kappa} .
\end{aligned}
$$

As a result,

$$
\begin{aligned}
j\left(u-u_{h}^{*}, u-u_{h}^{*}\right) & \leq \sum_{F \in \mathcal{F}} \frac{h_{F}^{2}}{p^{\frac{7}{2}}} \beta_{\infty, F}\left\|\left[\nabla\left(u-u_{h}^{*}\right) \cdot n\right]\right\|_{F}^{2} \\
& \leq c \sum_{\kappa \in \mathcal{K}} \frac{h_{\kappa}^{2}}{p^{\frac{7}{2}}}\left(p\left(\frac{h_{\kappa}}{p}\right)^{2 s-3}\|u\|_{s, \kappa}^{2}+p^{2} h_{\kappa}^{-1}\left\|\nabla\left(u-u_{h}^{*}\right)\right\|_{\kappa}^{2}\right),
\end{aligned}
$$

yielding (73).

(B) Since $\|v\|_{\varepsilon, j} \leq c(\varepsilon+1)\|v\|_{1, \Omega}+j(v, v)$, it is inferred using (73) that

$$
\left\|u-u_{h}^{*}\right\|_{\varepsilon, j} \leq c\left(\varepsilon^{\frac{1}{2}}+1+h^{\frac{1}{2}} p^{-\frac{3}{4}}\right)\left(\frac{h}{p}\right)^{s-1}\|u\|_{s, \Omega} .
$$

Owing to Lemmata 6.1 and 6.2 ,

$$
\begin{aligned}
\left\|u-u_{h}\right\|_{\varepsilon, j}^{2} & \leq a_{\varepsilon}\left(u-u_{h}, u-u_{h}^{*}\right)+j\left(u-u_{h}, u-u_{h}^{*}\right) \\
& \leq\left(1+\varepsilon^{-\frac{1}{2}}\right)\left\|u-u_{h}\right\|_{\varepsilon, j}\left\|u-u_{h}^{*}\right\|_{\varepsilon, j} .
\end{aligned}
$$


Hence,

$$
\begin{aligned}
\left\|u-u_{h}\right\|_{\varepsilon, j} & \leq\left(1+\varepsilon^{-\frac{1}{2}}\right)\left\|u-u_{h}^{*}\right\|_{\varepsilon, j} \\
& \leq\left(1+\varepsilon^{-\frac{1}{2}}\right)\left(\varepsilon^{\frac{1}{2}}+1+h^{\frac{1}{2}} p^{-\frac{3}{4}}\right)\left(\frac{h}{p}\right)^{s-1}\|u\|_{s, \Omega},
\end{aligned}
$$

yielding (70). The conclusion is straightforward.

Proposition 6.2 shows that in the diffusion-dominated regime, the $h p$-CIP bilinear form $j$ need not be modified to guarantee optimal convergence in $h$ and $p$. Furthermore, the above results indicate that the Péclet number to detect the regime transition is $\frac{h}{\varepsilon} p^{-\frac{3}{2}}$.

Remark 6.1. Following the ideas developed in [12, it is interesting to show that under mild additional assumptions on the approximability properties of a smooth subspace of the finite element space $V_{h}^{p}$, it is possible to derive in the diffusiondominated regime, both energy-norm and $L^{2}$-norm error estimates that are independent of the $h p$-CIP bilinear form $j$. Let $Z_{h}^{p}=V_{h}^{p} \cap C^{1}(\bar{\Omega})$ and assume that $Z_{h}^{p}$ is endowed with the following optimal $h p$-approximation property: There is $c$, independent of $p$ and $h$, such that for all $v \in H^{s}(\Omega)$, there is $z_{h}(v) \in Z_{h}^{p}$ such that

$$
\left\|v-z_{h}(v)\right\|_{1, \Omega} \leq c\left(\frac{h}{p}\right)^{s-1}\|v\|_{s, \Omega},
$$

where $s=\min (p+1, q) \geq 2$ and $q$ is defined in Proposition 6.2. Property (74) is expected to hold when the polynomial order $p$ is large enough. In the diffusiondominated regime $(\epsilon \sim 1)$, the bilinear form $a_{\epsilon}$ is $H^{1}$-coercive and bounded on $H^{1}(\Omega) \times H^{1}(\Omega)$ with constants that are of order unity. Furthermore, since $Z_{h}^{p} \subset$ $C^{1}(\bar{\Omega})$, the following improved consistency property holds: For all $z_{h} \in Z_{h}^{p}$,

$$
a_{\epsilon}\left(u-u_{h}, z_{h}\right)=j\left(u_{h}, z_{h}\right)=0,
$$

whence it is readily inferred using (74) that

$$
\left\|u-u_{h}\right\|_{1, \Omega} \leq c\left(\frac{h}{p}\right)^{s-1}\|u\|_{s, \Omega} .
$$

Moreover, assuming that problem (63) has elliptic regularity and using a standard Aubin-Nitsche duality argument yields

$$
\left\|u-u_{h}\right\|_{\Omega} \leq c\left(\frac{h}{p}\right)^{s}\|u\|_{s, \Omega} .
$$

The $h$-version of the above error estimates can be found in [12].

\section{Conclusions}

The $h p$-CIP finite element method analyzed in this paper can be used to approximate efficiently advection and (advection-dominated) advection-diffusion equations on tensor-product meshes. The error estimates are suboptimal by a factor of $p^{\frac{1}{4}}$ in the advection-dominated regime, while optimality is recovered in the diffusiondominated regime. In contrast, several questions remain open for simplicial finite elements. In particular, the existence of an admissible set of nodes yielding the best possible inverse trace inequality (48) and the sharpness of the estimates derived in 
Lemmata 5.3 and 5.4 deserve further investigation. Finally, it is worthwhile to mention that the design of an $h p$-CIP finite element method for the Oseen equation is straightforward by combining the above results with those of [8] and that numerical experiments in one space dimension, including convergence rates and estimates of condition numbers, are reported in [7].

\section{ACKNOWLEDGMENTS}

The authors are thankful to the anonymous referees for constructive comments and to Professor Ch. Schwab for stimulating discussions. This work was partly supported by the GdR MoMaS (CNRS-2439, ANDRA, BRGM, CEA, EdF).

\section{REFERENCES}

1. I. Babuška and M.R. Dorr, Error estimates for the combined $h$ and $p$ versions of the finite element method, Numer. Math. 37 (1981), no. 2, 257-277. MR623044 (82h:65080)

2. I. Babuška and M. Suri, The $h p$ version of the finite element method with quasi-uniform meshes, RAIRO, Math. Mod. Numer. Anal. 21 (1987), 199-238. MR0896241 (88d:65154)

3. I. Babuška and M. Zlámal, Nonconforming elements in the finite element method with penalty, SIAM J. Numer. Anal. 10 (1973), 863-875. MR0345432 (49:10168)

4. G.A. Baker, Finite element methods for elliptic equations using nonconforming elements, Math. Comp. 31 (1977), no. 137, 45-59. MR0431742 (55:4737)

5. E. Burman, A unified analysis for conforming and nonconforming stabilized finite element methods using interior penalty, SIAM J. Numer. Anal. 43 (2005), no. 5, 2012-2033 (electronic). MR2192329

6. E. Burman and A. Ern, Stabilized Galerkin approximation of convection-diffusion-reaction equations: discrete maximum principle and convergence, Math. Comp. 74 (2005), no. 252, 1637-1652 (electronic). MR 2164090 (2006e:65211)

7. _ Continuous interior penalty hp-finite element methods for transport operators, Numerical Mathematics and Advanced Applications (Berlin), Springer, 2006, Enumath 2005 Conf. Proc.

8. E. Burman, M. Fernández, and P. Hansbo, Continuous interior penalty finite element method for the Oseen's equations, SIAM J. Numer. Anal. 44 (2006), 1248-1274. MR2231863

9. E. Burman and P. Hansbo, Edge stabilization for Galerkin approximations of convectiondiffusion-reaction problems, Comput. Methods Appl. Mech. Engrg. 193 (2004), 1437-1453. MR2068903 (2005d:65186)

10. C. Canuto and A. Quarteroni, Approximation results for orthogonal polynomials in Sobolev spaces, Math. Comp. 38 (1982), 67-86. MR0637287 (82m:41003)

11. P.J. Davis and P. Rabinowitz, Methods of numerical integration, Computer Science and Applied Mathematics, Academic Press Inc., Orlando, FL, 1984. MR760629 (86d:65004)

12. J. Douglas Jr. and T. Dupont, Interior penalty procedures for elliptic and parabolic Galerkin methods, Computing Methods in Applied Sciences (Berlin) (R. Glowinski and J.-L. Lions, eds.), Lecture Notes in Physics, vol. 58, Springer-Verlag, 1976, pp. 207-216. MR440955 $(55: 13823)$

13. L. El Alaoui and A. Ern, Residual and hierarchical a posteriori error estimates for nonconforming mixed finite element methods, M2AN Math. Model. Numer. Anal. 38 (2004), no. 6, 903-929. MR2108938 (2006a:65147)

14. A. Ern and J.-L. Guermond, Discontinuous Galerkin methods for Friedrichs' systems. I. General theory, SIAM J. Numer. Anal. 44 (2006), 753-778. MR2218968

15. K. O. Friedrichs, Symmetric positive linear differential equations, Comm. Pure Appl. Math. 11 (1958), 333-418. MR0100718 (20:7147)

16. R.H.W. Hoppe and B. Wohlmuth, Element-oriented and edge-oriented local error estimators for non-conforming finite element methods, Math. Model. Numer. Anal. 30 (2) (1996), $237-$ 263. MR.1382112 (97e:65124)

17. P. Houston, D. Schötzau, and Th.P. Wihler, Energy norm a posteriori error estimation of hpadaptive Discontinuous Galerkin methods for elliptic problems, Tech. Report IMA Preprint Series 1985, Institute for Mathematics and its Applications, 2004. 
18. P. Houston, Ch. Schwab, and E. Süli, Stabilized hp-finite element methods for first-order hyperbolic problems, SIAM J. Numer. Anal. 37 (2000), no. 5, 1618-1643 (electronic). MR 1759909 (2001f:65135)

19. , Discontinuous hp-finite element methods for advection-diffusion-reaction problems, SIAM J. Numer. Anal. 39 (2002), no. 6, 2133-2163. MR1897953 (2003d:65108)

20. P. Houston and E. Süli, Stabilised hp-finite element approximation of partial differential equations with nonnegative characteristic form, Computing 66 (2001), no. 2, 99-119. MR1825801 (2002c:65211)

21. M. Jensen, Discontinuous Galerkin methods for Friedrichs systems with irregular solutions, Ph.D. thesis, University of Oxford, Oxford, UK, 2004.

22. O. Karakashian and F. Pascal, A posteriori error estimates for a discontinuous Galerkin approximation of second order elliptic problems, SIAM J. Numer. Anal. 41 (2003), no. 6, 2374-2399. MR2034620 (2005d:65192)

23. J. M. Melenk, hp-interpolation of nonsmooth functions and an application to $h p$-a posteriori error estimation, SIAM J. Numer. Anal. 43 (2005), no. 1, 127-155 (electronic). MR2177138

24. A. Quarteroni and A. Valli, Numerical approximation of partial differential equations, Springer Series in Computational Mathematics, vol. 23, Springer-Verlag, 1994. MR1299729 (95i:65005)

25. Ch. Schwab, $p$ - and hp-finite element methods, Numerical Mathematics and Scientific Computation, The Clarendon Press Oxford University Press, New York, 1998. MR1695813 (2000d:65003)

26. T. Warburton and J.S. Hesthaven, On the constants in $h p$-finite element trace inverse inequalities, Comput. Methods Appl. Mech. Engrg. 192 (2003), 2765-2773. MR1986022 (2004d:65146)

Institut D'Analyse et Calcul Scientifique (CMCS/IACS), Ecole Polytechnique Fédérale de Lausanne, Station 8, CH-1015 Lausanne, Switzerland

E-mail address: Erik.Burman@epfl.ch

Cermics, Ecole des Ponts, ParisTech, Champs-sur-Marne, 77455 Marne la Vallée, Cedex 2, France

E-mail address: ern@cermics.enpc.fr 